\title{
Monetary Attribute of Bitcoin
}

\author{
Chenghao Yang ${ }^{a}$, Yanwen Tian ${ }^{b}$
}

School of business, Renmin University of China, Beijing, China.

arbsyangchenghao@163.com, brbstianyanwen@163.com

\begin{abstract}
In In recent years, new virtual currency which is born on the Internet, has attracted more and more attention. Especially represented by the bitcoin, which is decentralized and has limited amount, has become active in the global market. Digital storage, decentralization, limited amount, complete anonymity and confidentiality, all make the strong monetary attribute. However, whether bitcoin, whose nickname is gold 2.0, could complete monetary attribute, and whether it could perfect the monetary functions, need to be researched and demonstrated. In addition, the public awareness of bitcoin isn't much. They don't realize the superiority and the shortcomings of bitcoin. In this paper, we start from the introduction of bitcoin, discuss the monetary attribute in detail, and analyze the problems and challenges which bitcoin faces. At last, make a summary, and discuss the meaning and the future.
\end{abstract}

Keywords: Monetary attribute, Hayek free currency theory, Credit money, General giving and receiving, Decentrar.

\section{浅论比特币的货币属性}

\author{
杨成浩, 田艳文 \\ 中国人民大学商学院, 北京, 中国
}

摘 要: 近年来, 诞生于互联网的新型虚拟货币吸引了越来越多的关注。尤其是以比特币为代 表的去中心化、有限总量的虚拟货币, 开始活跃于全球市场。数字化存储、去中心化、数量 有限、完全匿名、高度加密等特征，共同赋予比特币极强的货币属性。但是，被称为黄金2.0 的比特币是否具有完备的货币属性, 又是否能完美履行各项货币职能, 这是有待研究和论证 的。除此之外, 大众对比特币的认识尚浅, 并没有真正认识到比特币作为新型虚拟货币的优 越性和相关缺陷。本文从比特币的介绍出发, 详细论述比特币的货币属性, 分析比特币目前 面临的问题和挑战，最后简单总结，论述比特币的意义和未来前景。

关键词：货币属性、哈耶克自由货币理论、信用货币、一般授受性、去中心化

\section{1. 前言}

\section{1 研究背景}

自 2013 年起，比特币就在中国飞速发展。区块链、椭圆曲线数字签名算法 ECDSA、Hash 哈希 算法、矿机和矿池等比特币相关专业术语逐渐进入人们视野。比特币的币值在波动中却又飞 速攀升的同时, 中国比特币市场一度拥有了全球 95\%的交易量, 以及控制超过 50\%的算力。除 此之外，各类新的虚拟货币层出不穷，比如莱特币（Litecoin），同样是点对点加密货币， 去中心化，并且在比特币的基础上实现了不少的改进。

就在全球虚拟货币市场日趋火热时， 2017 年 4 月，比特币勒索病毒 “永恒之蓝” 席卷全球， 造成了严重影响, 并在一定程度上挫伤了比特币交易市场。不久, 中国政府在 9 月 4 日正式 发文，严令禁止 ICO，国内的比特币交易平台也相继关停，中国比特币交易市场宣告落幕。 可与之同时，国外比特币市场却仍兴盛。日本政府在 4 月份出台了关于承认比特币合法的相 
关法案，随后在 12 月 11 日，芝加哥期权交易所（CBOE）正式宣布推出比特币期货，这标志 着美国把比特币纳入主流金融体系。

从 2009 年比特币诞生, 比特币的发展可以说是历经波折, 各国对于比特币的态度也莫衷一是。 对于比特币未来的发展，更是充满着未知与挑战。

\section{2 研究意义}

比特币的迅速发展以及在国际市场上的起起落落, 吸引了各界人士的关注。但是以比特币作 为典型代表的虚拟货币, 是否具有相应的货币属性, 能否履行货币的各项职能, 甚至超越目 前以国家信用为基础、具有中心化特点的 “传统货币”，这需要进一步的研究和论证。 其中最为重要的是，普罗大众对于虚拟货币的认识尚浅，误解较多。本文从比特币的发展历 程出发, 详细讨论比特币的货币属性以及分析比特币的优劣势, 简单总结比特币目前面临的 问题和未来发展的方向，最终起到认识比特币的参考作用。

\section{3 国外文献综述}

国外对比特币等虚拟货币的研究文献较多，包括对比特币的性质界定以及实证方面的研究。 尤其在比特币的性质界定上, 有着较大的争论。公众难以界定比特币的属性, 可能将其归类 为一种货币、商品或者服务。

2013 年，比特币已经在德国被描述为“另一种形式的货币”或“私有货币”。而Wallace (2011) 认为比特币已经成为国际间货币一种廉价交换方式。除此之外, Chowdhury 和 Mendelson （2014）在虚拟货币和金融体系的框架下，认为人们对于比特币类的虚拟货币的认可程度需 要虚拟货币不断发现其内在价值作为依据，但比特币类的虚拟货币成为主流货币只是时间问 题。Woo 等人 (2013) 也讨论了比特币的内在价值, 并对比特币的未来持乐观态度。但他们 同时指出，市场投机行为可能造成比特币汇率大起大落，进而影响其被社会接受的程度。

Surda（2012）认为比特币的价值只是取决于市场力量和使用者信心，比特币的价值是没有保 证的, 所以比特币与其说是一种货币, 不如说是一种商品。Philipp Guring 与 Ian Grigg 认 为在挖矿成本可被控制的情况下, 比特币经济容易发生崩塌。而 Yermack（2013）直接认为 比特币是一种投机性工具。

\section{4 国内文献综述}

国内学者对于比特币的态度也莫衷一是。

杨晓晨，张明（2014）认为比特币的出现是解决当前国别货币面临问题的积极尝试，并针对 交易数据对比特币市场典型特征作出了初步实证分析。贾丽平（2013）[1]也积极地指出, 比 特币作为一种全新的无政府虚拟货币，一场数字货币的革新，代表了世界政治经济一体化思 潮，具备一定的革命性。除此之外，王素珍（2014）[2]指出在未来，比特币将通过挂钩法定 货币最终成为跨主权的世界货币。

但同时, 盛松成和张璇（2014）[3]旗帜鲜明地指出, 以比特币为代表的虚拟货币本质上不是 货币。孙皓原和杨祖艳（2016）[4] 也详细说明了比特币在流通手段、价值尺度和价值穴藏上 的不完备货币属性。

\section{2 比特币的性质}

\section{1 简述比特币}

2008 年全球爆发金融危机, 各国政府和银行协调经济发展的能力和信誉受到了社会各界的极 大质疑, 同年 11 月 1 日, 中本聪 (Satoshi Nakamoto) 先生在互联网上发表了研讨陈述,

《Bitcoin_A_Peer-to-Peer_Electronic_Cash_System》，陈述对电子货币的新设想。比特币 并于 2009 年实现, 进入现实世界。从 2009 年比特币诞生, 到 2017 年, 短短八年间, 比特币 的币值发生了出人意料的增长和频繁的波动。

比特币，作为 “去中心化” 的电子货币，没有集中的发行方，每个货币均由网络节点的计算 生成, 每个人都能通过运行一个自由的开放源代码软件来获取比特币。因此, 比特币系统是 一个平等的点对点 (P2P) 系统, 即比特币的去中心化或者自组织系统（DAC, Distributed 
Autonomous Corporation）。所有交易者通过数字代码同时实现交易的支付、计价和价值储 藏功能。比特币利用椭圆曲线数字签名算法 ECDSA 双重加密, 安全性极高, 并且其货币总量 拥有最终上限，不能无限增长。除此之外，比特币的流通不需要中间机构，交易成本尤其是 跨国交易成本较低。根据王巍（2014）所言，比特币是自组织的数字支付体系，在互联网和 现实市场空间中具有广泛的货币替代功能，是重要的金融创新领域。

比特币的上述特征是由公认的数学公式提供保证，不由任何人、组织或者国家提供保证或控 制。换句话说，法定货币由国家信用背书，而比特币由自然科学理论提供证明和保障。

比特币的所具有的货币潜质, 比如去中心化、总量有限等特点, 可以与人类历史上广泛使用 的货币一一黄金相媲美。并且，比特币在某些方面比黄金更加理想，比如流通成本更低、流 通范围更广泛、可以被无限分割等。因此，比特币常被称为黄金 2.0 , 寄寓了人们对比特币 未来承担世界货币角色的期盼。

2.2 比特币的货币属性

货币是从商品中分离出来的特殊商品, 是商品交换的媒介, 而马克思指出： “在商品世界起 一般等价物的作用就成了货币特有的社会职能，从而成了它的社会独占权”，即货币的本质 是一般等价物，是价值尺度和流通手段的统一，并且具有价值尺度、流通手段、支付手段、 败藏手段和世界货币的五大职能。

价值尺度，亦称价值标准，赋予交易对象以价格形态。利用价值尺度，可以得到各类商品的 货币价格，也使得所有商品可以在这统一的标准下进行价值衡量、价值比较乃至商品交换。 比特币效仿黄金去中心化、有限产出的特点，成为具有稀缺性的虚拟货币，是网络中的数字 黄金，也逐渐被应用于各个领域，发挥价值尺度的社会职能。

流通手段，是指货币充当商品交换的媒介，人们可以通过使用货币，实现商品的交换。与法 定货币相比，比特币同样地发挥着交易媒介的功能，并且具有更强的分割能力，方便微型支 付。1 比特币最多可以分解为 1 亿份, 即 10 的 8 次方份 (最小面额 $0.00000001 B T C=1$ Satoshi)。 支付手段主要指桧销㻌购情况下, 货币发挥的延期交付作用, 是货币交易媒介职能的延伸, 满足了商业生产和生活的客观需要, 实现未来货币与财富的兄现。

败藏手段指货币在退出流通领域后发挥的社会财富积累、保值的职能, 发挥财富蓄水池的咜 藏作用。比特币在新型加密技术和互联网通信技术的两重加持下, 人们能更加安全、便捷地 陉藏货币，进一步实现财富的积累和保值。除此之外，比特币的有限总量可以避免由于政府 滥发货币造成的通货膨胀。

世界货币，则是指货币跨越国家界限，在国际市场上发挥一般等价物的作用，以达成国际贸 易。比特币在网络 P2P 支付条件下，不需要经过中介机构，极大地减少了国际交易的手续和 成本, 减少了汇率损失, 更提高了国际贸易的效率。所以比特币在世界货币职能的发挥上, 拥有更大的优势。

从 2010 年美国程序员拉斯洛第一次使用比特币购买披萨饼，到服饰领域（Bitfash.com）的 比特币应用, 再到汽车、硬通货、教育领域, 甚至商业航天公司维珍银河（Virgin Galactic） 在 2013 年也开始接受比特币支付太空旅行。比特币逐渐渗透了人类生活的各个领域, 越来越 多的国家和地区也开始接受比特币。

正如贾丽平 [1]所说, 比特币是信用货币进化的阶段性产物, 反映了信息对称、个体选择、去 中心化和权力制衡的货币特征，映射了货币由分散性向单一性回归的进化需求。但同时，她 也强调，比特币基本上具有货币的功能，拥有货币的部分属性，但不一定是真正的货币。它 未来能否过渡为真正货币取决于人们对比特币的信任、对一整套机制所营造的信心。简而言 之，比特币的货币属性尚不完备，其所面临的问题和挑战也较多。 


\section{3 比特币面临的问题与挑战}

3.1 比特币尚不完备的货币属性

首先在价值尺度上，由于比特币的 “去中心化” 特征，即比特币没有集中的发行和调节机构， 不同于拥有中央调节机制的现代信用货币体系。因此，比特币币值的波动难以被调节，尤其 是存在外部炒作时，币值波动难以尉平，币值的剧烈波动又提供了巨大套利空间，进一步推 动过度炒作, 形成恶性循环 [3]。比特币从 2011 年初 0.3 美元上涨至 2013 年 11 月的最 高 1300 美元，然后又迅速回落至 2015 年初的最低 200 美元左右。而就在 2017 年 12 月 11 日，芝加哥期权交易所正式上线比特币期货，比特币的价格达到 16500 美元/枚，突破 10 万 元人民币，年初至今涨幅近 1919\%。价值相对稳定是一种货币履行价值尺度和流通手段职能 的基本前提，而比特币近年频繁且巨大的币值波动，使其在作为价值标准方面遭遇着巨大的 挑战。

其次，比特币的有限总量性加强了个人对比特币币值升值的心理预期，使得人们更愿意将比 特币作为投资品甚至投机品，而不是作为经济生活中的交换媒介。据 Yermack（2013）的 统计评估，比特币交易每天发生约 7 万笔，其中 $80 \%$ 是投资或投机行为，仅有 1.5 万次可 以看做购买商品和服务, 相比于全球日均实际交易规模, 比特币作为交易媒介的作用发挥极 为有限。长此以往, 比特币将逐渐退出流通市场, 失去货币流通手段的职能。

在价值尺度和流通手段衍生而来的支付手段、咜藏手段和世界货币职能方面，比特币同样面 临着诸多问题。比如比特币币值的巨大波动, 风险甚至大于许多风险资产，其价值它藏职能 被大打折扣，目前更适合被作为投机资产。除此之外，各国政府对比特币莫衷一是的态度， 尤其是个别政府对比特币交易的禁令，极大影响比特币行使世界货币职能。

简而言之, 比特币目前的货币属性尚不完备, 不能很好地履行货币的各项职能, 因而并不具 备替代传统信用货币的能力。

\section{2 比特币面临的其他问题和挑战}

不仅在货币属性方面，比特币在其他方面也面临着诸多的问题。

去中心化的特征使得比特币几乎摆脱了一切人为调控, 避免了货币滥发导致的通货膨胀, 但 与之对应，政府失去了对经济的重要调节手段。同时，比特币有限的数量与不断扩大的社会 生产和商品流通之间存在矛盾, 若成为本位币, 必然导致通货紧缩, 抑制经济发展。货币供 给应与经济社会的发展相适应，如果货币供给无法满足货币需求，即会极大地抑制商品流通 和社会生产的发展，甚至导致严重的通货紧缩，给经济发展带来更大危害。这也是金本位制 崩溃的根本原因。

另外, 孙皓原和杨祖艳 [4]指出非法定数字货币, 即比特币可以形成价格稳定的静态均衡和价 格不稳定的动态均衡。但具有内在的通货膨胀自我实现性质, 依靠该类货币发行机制无助于 从根本上解决通货膨胀问题。

哈耶克（1999）所强调的竞争性货币发行机制，是如今许多虚拟货币的起点，但是比特币暂 时没能满足购买力稳定等理论要求, 因而还不能很好地解决货币问题。除此之外, 虽然比特 币自身安全性极强，但是与之配套的交易软件、交易平台依然面临诸多的安全保障问题。配 套体系的不完善, 同样对比特币的推广应用有巨大冲击。2014 年 2 月, 全球最大的比特币交 易平台 Mt. Gox，就因为被黑客盗取巨额比特币而最终宣告破产。

\section{4 结论}

货币之所以成为货币，本质来源于人们对其的信心，也称之为一般授受性。尽管比特币拥有 货币相关的巨大潜质，可以在一定程度上履行起货币职能，但就目前而言，比特币的货币属 性尚不完备，在通货紧缩、安全使用等方面同样面临着巨大挑战。

比特币所具有的优于传统信用货币的特点, 是货币政策制定者需要关注和重视的, 需要在原 有信用货币基础上，通过更加合理的货币政策，更好地规避货币风险，使货币更加有效地推 
动社会经济生产的发展。同时, 以比特币为代表的诸多新型虚拟货币, 更需要不断改进, 克 服目前存在的问题，使其能够更加完美地履行货币职能。

正如达鸿飞所说，比特币是一场运动、一种思想。比特币已经重新定义了货币，也将颠覆人 类对货币的认识。这种思想就像一颗种子，一颗可以在每个人心里种下的种子。它种下的时 候也许无人知晓, 它发芽的时候也许毫不起眼, 但当种子渐渐长大, 在每个人心里都有一棵 大树的时候，它就具备了巨大的改革力量。

比特币只是货币改革的起点, 绝不会是终点。未来货币一定能更加完美地推动经济发展, 当 下的诸多问题也将解决。

\section{References}

[1]. Liping Jia. The theory, practice and influence of bitcoin[J]. Studies of International Finance,2013,(12):14-25.

[2]. Suzheng Wang. Look at bitcoin in terms of currency[J]. China Finance,2014,(09):16-18.

[3]. Songcheng Sheng,Xuan Zhang. Virtual money is not a currency in essence -- in bitcoin, for example, practice and influence[J]. China Finance,2014,(01):35-37.

[4]. Haoyuan Sun,Zuyan yang. Research on non-legal digital currency competitiveness - based on full competitive market.[J]. Shanghai Finance, 2016, (09):27-34. 